Classification

Physics Abstracts

$64.70 \mathrm{M}-6130 \mathrm{G}-4280 \mathrm{~K}$

\title{
Linear electroclinic coupling in ferroelectric liquid crystals
}

\author{
L Dupont, M. Glogarová $\left(^{*}\right)$, J P Marcerou, H. T Nguyen, C Destrade and \\ L Lejček (*)
}

Centre de recherches Paul Pascal, Château Brvazac, Av Dr Schwettzer, F-33600 Pessac, France

(Recetved 16 January 1991, revised 08 March 1991, accepted 19 March 1991)

\begin{abstract}
Résumé. - Nous avons étudié le couplage électroclinıque (mode mou) près de la transition de phase smectıque A-smectıque $\mathrm{C}^{*}$ pour dıfférents composés chıraux La prıncıpale onginalité de notre montage est de permettre la mesure des quantıtés optıques et diélectrques dans un même échantıllon Nous pouvons aınsı déterminer les premiers coefficients du developpement de Landau associé à la transition de phase Les prédictions les plus simples de cette théorie sont verıfiées sans dépendances thermiques anormales des coefficients D'un autre point de vue, celus des applications, la frequence de relaxation de leffet électroclınique est de rordre de $10 \mathrm{kHz}$ à $1 \mathrm{MHz}$ pour les composés étudiés
\end{abstract}

\begin{abstract}
We report a study of the electroclinic coupling (soft mode), close to the smectic A to smectic $C^{*}$ phase transition in several chiral compounds The main characteristic of the technique used is to measure both optical and dielectric quantities on a single sample This way one may derive the first coefficients of the Landau expansion describing the phase transition The simplest expectations of this theory are found to hold well without unusual temperature dependences of the coefficients Furthermore, the relaxation frequency of the electroclinic coupling, (which is essential for applications), is found to lie in the $10 \mathrm{kHz}$ to $1 \mathrm{MHz}$ range
\end{abstract}

\section{Introduction.}

Ferroelectric smectıc $C^{*}$ liquid crystals have great potentialities in display technology as they may be used to build bistable and fast nonlinear devices $[1,2]$ ( $\mathrm{e}$ they are insensitive to weak electric fields in multiplexed displays while they switch quickly when suitably addressed) Another promising property of these materials has been pointed out [3,4], namely their great soft mode susceptibility in response to an external electric field (electroclinic coupling), in the high temperature smectic A phase, which can be used to design fast linear optical devices [5].

In this paper we report a study on the electroclinic coupling that allows the measurement of both optical and dielectric properties under the same conditions of temperature, applied electric field and aligninent in a given sample [6] This expenmental technique gives the first coefficients of the Landau expansion together with the soft mode viscosity and relaxation frequency

(*) Permanent address Institute of Physics, Czechoslovak Academy of Sciences, Na slovance 2, 18040 Prague 8, Czechoslovakıa 
Section 2 describes the experimental quantities we measure, while section 3 reports on the results we obtained for three different compounds An appendix reports theoretical expectations derived from the use of a general onentational order parameter.

\section{Theoretical background.}

21 ORDER PARAMETERS OF ORIENTATIONAL PHASE TRANSITIONS - The Smectic ASmectic C (or ferroelectric $C^{*}$ ) phase transition is described [7] by a two-dimensional order parameter $\theta \mathrm{e}^{i \phi}$ that measures the tilt of the director $\mathbf{n}$ at an angle $\theta$ with respect to the layer normal $z$, with a projection in the layer plane $x y$ at an angle $\phi$ from $x$ Another notation has been used [8] that takes into account the tensorial character of the two components of the order parameter :

$$
\begin{aligned}
& \theta_{x}=\theta \cos \phi=n_{x} \times n_{z}=\xi_{1} \\
& \theta_{y}=\theta \sin \phi=n_{y} \times n_{z}=\xi_{2} .
\end{aligned}
$$

This shows that, in order to depict the onentational phase transitions from a uniaxial phase like a smectic $A$ to a biaxial one, smectic $C$ or $C^{*}$ or biaxial smectic $A$ [9], one has to use a tensorial order parameter measuring the deviations of a tensor like the dielectric permittivity $\varepsilon_{i j}$ or the magnetic susceptibility $\chi_{i j}$ out of its equilibrium uniaxial value This tensor may be defined as

$$
Q_{1 \jmath}=\varepsilon_{\imath \jmath}-\varepsilon_{\imath \jmath}^{0}
$$

With $Q_{l}$ one may build, like we do in the appendix, a Landau free energy density [10] obeying the symmetry of the problem This will be useful for the study of different problems which lie out of the scope of this paper, like transverse biaxiality or the occurrence of first order smectic A-smectic $\mathrm{C}^{*}$ phase transition For the study we report here, the tilt angle $\theta$ will be used

22 LINEAR ELECTROCLINIC EFFECT (SOFT MODE) IN THE SMECTIC A PHASE - The expression (14) developed in appendix for the free energy $F^{*}$ may be taken as a starting point for the study of the smectic A-smectic $\mathrm{C}^{*}$ phase-transition with $\left(Q_{x z}, Q_{y z}\right)$ playing the role of the primary order parameter This is still an open field [11-13] we will not discuss, as we are interested here in the coupling of this order parameter with an applied electric field. This coupling is strong enough $[13,14]$ to induce tilt angles of a few degrees in the smectic A phase close to the transition so we will limit ourselves to the linear regime where $\theta$ is proportional to the applied field. We assume that the samples are in the ideal SSFLC configuration [2], where the spontaneous splay and twist of the director are removed by surface interactions. Last, the electric field $E$ and the polarization $P$ have a fixed direction in the plane of the layers determining the $C_{2}$ axis of the induced smectic $C$ phase

Under all these conditions, the first line of equation (14) developed up to fourth order in $\theta$, with the addition of the applied electric field $E$ yields

$$
F^{*}=\frac{1}{2} B_{\perp} \theta^{2}+\frac{1}{2 \varepsilon_{0} X_{\perp}} P^{2}-c P \theta+\frac{1}{4} b \theta^{4}-P \cdot E-\frac{\varepsilon_{\infty}}{2} E^{2}
$$

where $B_{\perp}=\alpha\left(T-T_{\mathrm{c}}\right), \alpha>0$ and $\varepsilon_{\infty}=\varepsilon_{0}\left(1+\chi_{\infty}\right)=n_{0}^{2}$ contains high frequency molecular librations and electronic contributions to $\varepsilon_{\perp}$.

Minimization of $F^{*}$ leads to $\theta$ and $P$ proportional to the applied field $E$

$$
\begin{aligned}
B_{\perp} \theta-c P & =0 \\
-\varepsilon_{0} \chi_{\perp} c \theta+P & =\varepsilon_{0} \chi_{\perp} E
\end{aligned}
$$


We shall call hereafter this situation the linear regime which will be valid provided that the quartic term $\theta^{4}$ may be neglected with respect to the quadratic one $\theta^{2}$ in $F^{*}$ In the mean field approximation $\theta$ and $P$ in smectic $A$ phase read:

$$
\theta=\frac{\varepsilon_{0} \chi_{\perp} c}{B_{\perp}^{*}} E, \quad P=\varepsilon_{0} \chi_{\perp} E+\frac{\varepsilon_{0}^{2} \chi_{\perp}^{2} c^{2}}{B_{\perp}^{*}} E,
$$

where

$$
B_{\perp}^{*}=\alpha\left(T-T_{\mathrm{c}}^{*}\right), \quad T_{\mathrm{c}}^{*}=T_{\mathrm{c}}+\frac{\varepsilon_{0} \chi_{\perp} c^{2}}{\alpha}
$$

One finds that the transition temperature is displaced in a chiral compound, due to the linear coupling $c P \theta$, as proved experimentally by Bahr [14] and recently checked [15] for one of the compounds we report on in this paper (C7 Valine) mixed with its enantiomer in varied proportions From a dynamical point of view, if the applied field is a sine one $E \mathrm{e}^{j \omega \prime}, B_{\perp}^{*}$ is to be replaced by $B_{\perp}^{*}+j \omega \gamma_{\theta}$, where $\gamma_{\theta}$ is the soft mode viscosity Then one expects the frequency dependence of $\theta$ and of the electroclinic component in $P$ to be a Lorentzian with a relaxation frequency $B_{\perp}^{*} / 2 \pi \gamma_{\theta}$ proportional to $\left(T-T_{c}^{*}\right)^{-1}$

If we now express the linearity condition $2 B_{\perp}^{*} \theta^{2} \gg b \theta^{4}$, we get :

$$
E \ll \sqrt{\frac{2 B_{\perp}^{* 3}}{b \varepsilon_{0}^{2} \chi_{\perp}^{2} c^{2}}} \propto\left(T-T_{c}^{*}\right)^{3 / 2}
$$

This relation, checked carefully as reported in figure 3, means that when studying the electroclinic effect in its linear regime, one has to apply an increasingly small electric field when approaching the phase transition It expresses nothing but the usual definition of a susceptibility close to a phase transition

$$
\frac{\varepsilon_{0} \chi_{\perp} c}{B_{\perp}^{*}}=\lim _{E \rightarrow 0} \frac{\theta}{E}
$$

23 SOFT MODE IN THE SMECTIC C* PHASE - In the condensed smectic C* phase, the soft mode linked to the modulus of the order parameter $\theta$ is often hidden by the Goldstone mode linked to the phase $\phi$. It is still present however as a perturbation $\delta \theta$ of the equilibrium value $\theta_{0}$. There also exists a perturbation $\delta P_{\mathrm{ec}}$ of the permanent polarization. They may be written in the linear regime, for a second order phase transition, as:

$$
\begin{aligned}
\delta \theta & =\frac{\varepsilon_{0} \chi_{\perp} c E}{2 B_{\perp}^{*}+J \omega \gamma_{\theta}} \\
\delta P_{\mathrm{ec}} & =\frac{\varepsilon_{0}^{2} \chi_{\perp}^{2} c^{2} E}{2 B_{\perp}^{*}+J \omega \gamma_{\theta}}
\end{aligned}
$$

One recovers a Curie-Weiss law with a factor of 2 between the amplitudes of $\delta \theta$ and $\delta P_{\text {ec }}$ in the smectic $\mathrm{A}$ and $\mathrm{C}^{*}$ phases In a similar way, the relaxation frequency in the condensed phase is twice the high symmetry one, at the same distance from $T_{\mathrm{c}}^{*}$

The same caution concerning the amplitude of the electric field applies here, showing that in thick samples where one has to apply a bias field [16] in order to unwind the smectic $C^{*}$ helix, the interpretation of the experiments should be difficult due to the non linearity of the problem 


\section{Experimental study of the linear electroclinic effect.}

Our aim is to measure, for a given compound or mixture in a single SSFLC ${ }^{2}$ sample, the tilt angle $\theta$ and the polarization $P$ as a function of the applied electric field $E$, angular frequency $\omega$ and temperature $T$ We developed the set-up described in figure 1 in which the transmitted light is detected by a PIN photodiode from Hamamatsu giving a signal proportional to $\theta$ while the electric current in the cell is measured independently with the help of a Tektronix A6302 current probe. Both signals are measured by a lock-in amplifier from E.G G. (model 5206 up to $200 \mathrm{kHz}$ and model 5202 from $100 \mathrm{kHz}$ to the $\mathrm{MHz}$ range) The temperature is regulated with a stability of about $002 \mathrm{~K}$. The studied compounds and their phase sequences are listed in table I

The samples were aligned in the bookshelf SSFLC planar geometry [2] with a thickness of about $2 \mu \mathrm{m}$ obtained without spacers for the Isoleucine compounds and a thickness of $15 \mu \mathrm{m}$ with spacers for the C7 Valine compound. Without spacer, one minimizes the occurrence of chevrons [17]. but the exact thickness of the sample and consequently the applied field are poorly known One must notice that such thin planar smectic A samples show penodic

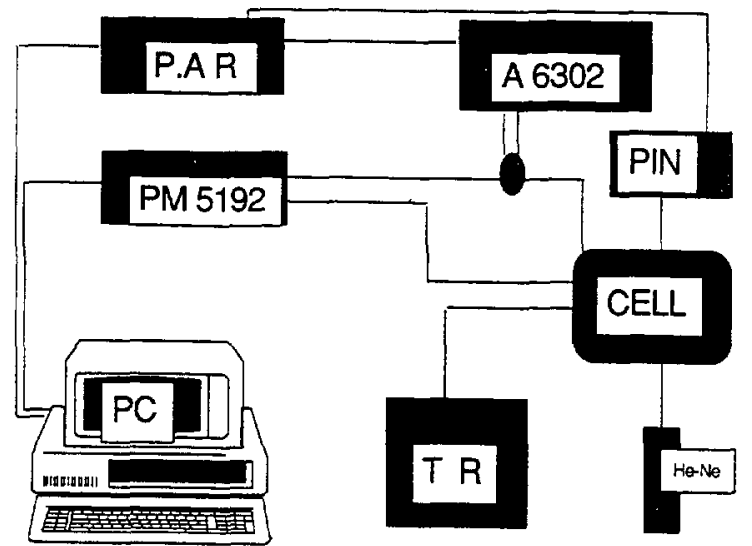

Fig 1 - Experimental set-up PAR lock-in amplifier, He-Ne laser, PIN : photodiode, A6302 current probe, Cell two stages oven, PM5192 frequency synthesizer, PC microcomputer, TR thermal regulation

Table I - Studied compounds (the smectic phases in parentheses are monotropic).

\begin{tabular}{|c|c|c|c|}
\hline Name & & Formula & Phase sequence \\
\hline $\begin{array}{l}\text { COS-C10 } \\
\text { Isoleucine }\end{array}$ & I & $\begin{array}{l}\mathrm{C}_{10} \mathrm{H}_{21} \mathrm{O} \bigcirc \bigcirc \mathrm{COS} \bigcirc \bigcirc \\
\mathrm{O}_{2} \mathrm{C}-\mathrm{C}^{*} \mathrm{HClC} \mathrm{HCH}_{3} \mathrm{C}_{2} \mathrm{H}_{5}\end{array}$ & $\begin{array}{l}\text { Cryst }-\mathrm{SC}^{*} \ldots \mathrm{SA}-\mathrm{I} \\
58^{\circ} \mathrm{C} \quad 66^{\circ} \mathrm{C} \quad 74^{\circ} \mathrm{C}\end{array}$ \\
\hline $\begin{array}{l}\text { C6 } \\
\text { Isoleucine }\end{array}$ & II & $\mathrm{C}_{6} \mathrm{HClC}_{13} \mathrm{O}-\bigcirc \mathrm{HCH}_{3} \mathrm{C}_{2} \mathrm{H}_{5}$ & $\begin{array}{l}\text { Cryst -- SC } \text { S }^{*} \text { SA - I } \\
47^{\circ} \mathrm{C} \quad 535^{\circ} \mathrm{C} \quad 61{ }^{\circ} \mathrm{C}\end{array}$ \\
\hline C7 Valıne & III & $\begin{array}{l}\mathrm{C}_{7} \mathrm{H}_{15} \mathrm{O} \bigcirc \bigcirc \mathrm{HClCH}\left(\mathrm{CH}_{3}\right)_{2} \\
\mathrm{C}^{*} \mathrm{HC}\end{array}$ & $\begin{array}{l}(\mathrm{SG})--\left(\mathrm{SC}^{*}\right)-\mathrm{SA}-\mathrm{I} \\
703^{\circ} \mathrm{C} \quad 74.1^{\circ} \mathrm{C} \quad 81^{\circ} \mathrm{C}\end{array}$ \\
\hline
\end{tabular}


patterns which have been attributed to chevron structure [18] or elongated parabolic focal conics, that are favored in chiral compounds when one applies an electric field [19] This superstructure was always present in the study we report here, and probably in all electroclinic experiments, so one must be aware that the accuracy of the quantitative tilt angle measurements is to be estimated at $\pm 10 \%$, while relaxation frequency is more precise This is a limitation common to all electroclinic experiments made with thin $(\approx$ some $\mu \mathrm{m})$ planar samples

31 Tilt angle MEASUREMENT - As usual [20], we work in the linear regime of the light detection between crossed polarizers, the optical axis making at rest a $\pi / 8$ angle with one of them In the limit of small deviation angles, the intensity of the transmitted hight is proportional to the tilt angle and is modulated at the same frequency as the applied electric field

$$
I\left(\frac{\pi}{8}+\delta \theta\right)=I\left(\frac{\pi}{8}\right) \times\left(1+4 \delta \theta \mathrm{e}^{j \omega t}\right)
$$

According to the previous discussion, $\delta \theta$ must obey in the smectic $\mathrm{A}$ and smectic $\mathrm{C}^{*}$ phases respectively

$$
\begin{aligned}
& \delta \theta\left(T>T_{c}^{*}\right)=\frac{\varepsilon_{0} \chi_{\perp} c E}{B_{\perp}^{*}+\jmath \omega \gamma_{\theta}}, \\
& \delta \theta\left(T<T_{c}^{*}\right)=\frac{\varepsilon_{0} \chi_{\perp} c E}{2 B_{\perp}^{*}+\jmath \omega \gamma_{\theta}}
\end{aligned}
$$

where $B_{\perp}^{*}$ is in first approximation [21] the only temperature dependent coefficient, $B_{\perp}^{*}=\alpha\left(T-T_{\mathrm{c}}^{*}\right)$ in the mean field approximation or $\alpha\left(T-T_{\mathrm{c}}^{*}\right)^{\gamma}$ otherwise The first step of our experimental procedure consists in the determination of the maximum value $E_{\mathrm{max}}$ of the electric field for which the response is still linear ( $\left.F_{1} g 2\right)$

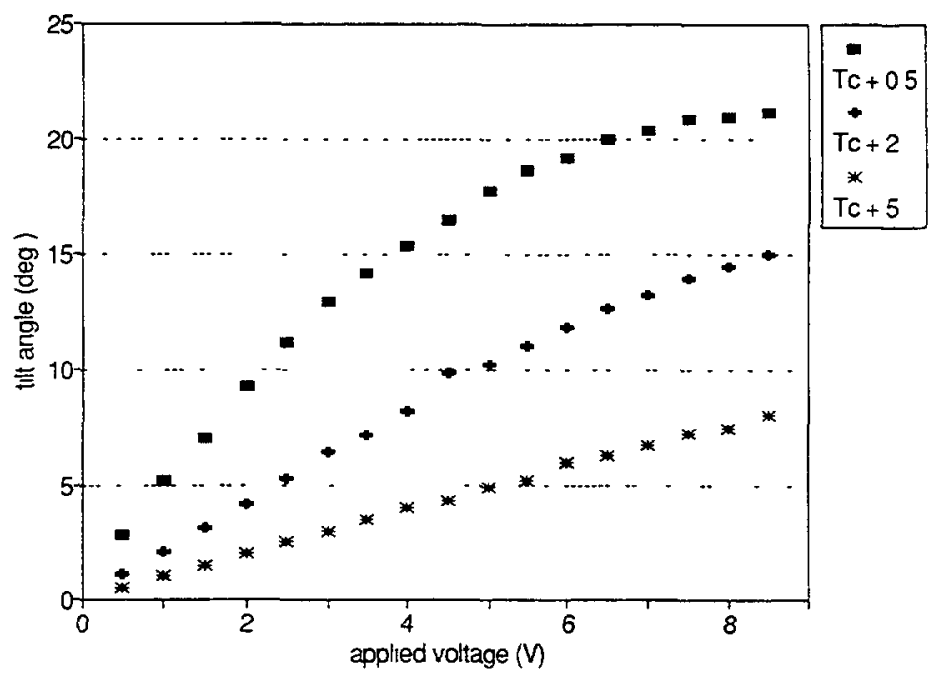

Fig 2 - Plot of the angle $\theta$ as a function of the applied voltage for different temperatures in a $2 \mu \mathrm{m}$ thick COSC10 Isoleucine sample 


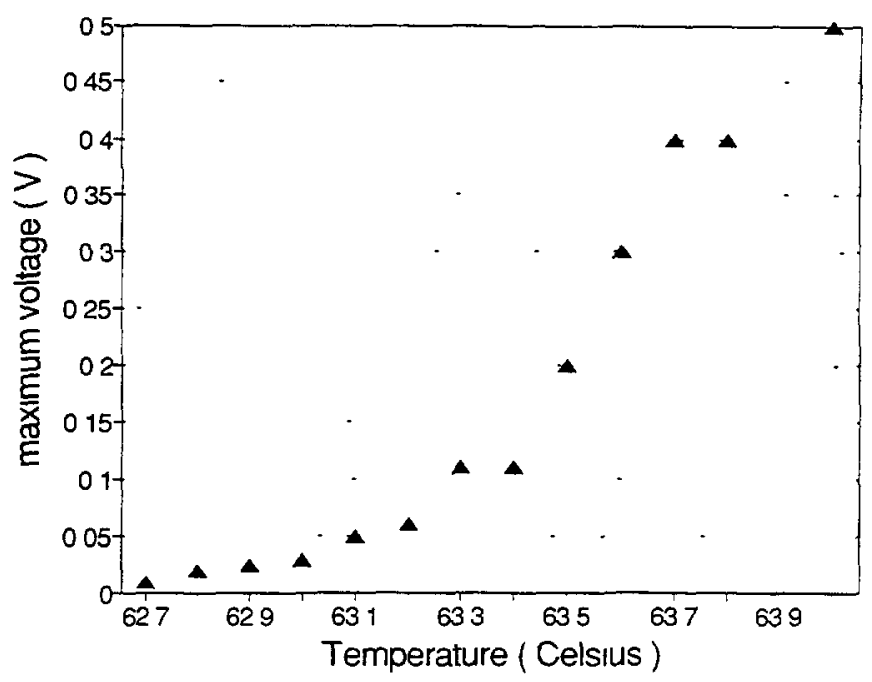

Fig 3 - Maximum applied voltage in smectic A phase of a $2 \mu \mathrm{m}$ thick COSC 10 Isoleucine sample as a function of temperature in agreement with the linearity condition expressed by equation (10)

If one plots this maximum $E_{\max }$ as a function of temperature, one recovers a curve (Fig 3 ) in agreement with equation (6) where the applied voltage has to be as low as $50 \mathrm{mV}$ rms in a $2 \mu \mathrm{m}$ thick sample close to $T_{c}^{*}$

$$
\left(T-T_{\mathrm{c}}^{*} \approx 05 \mathrm{~K}\right)
$$

The second step of our expenmental procedure consists then in recording at different temperatures the frequency dependence of the tilt angle in order to recover the expected Lorentzian law $(\theta(\omega)$ in Fig 4 and doubly logarithmic plot in Fig 5)

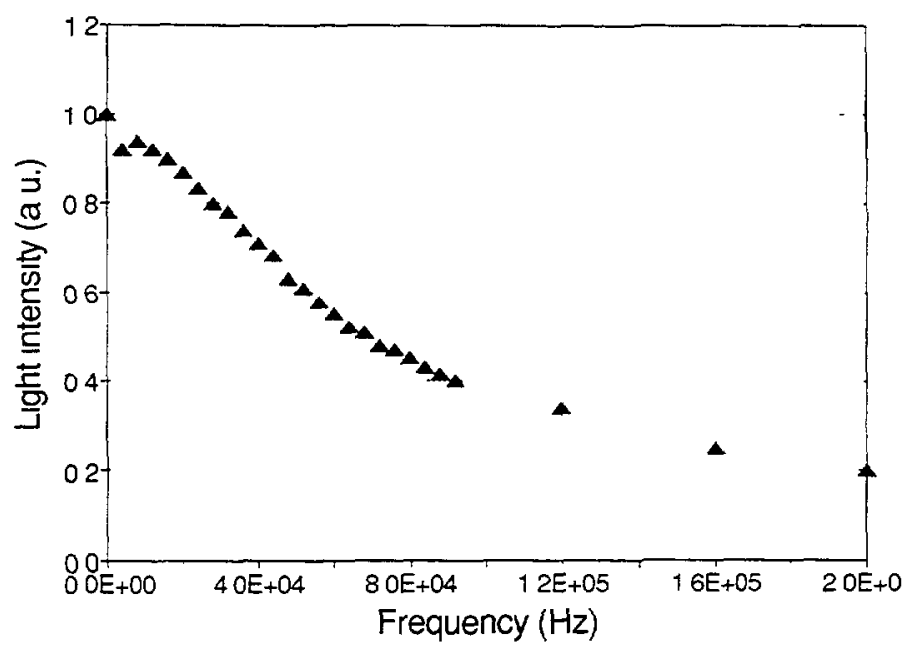

Fig 4 - Variation of light intensity proportional to ult angle modulation in COSC10 Isoleucine at $64{ }^{\circ} \mathrm{C}\left(\approx T_{L}+1{ }^{\circ} \mathrm{C}\right)$ 


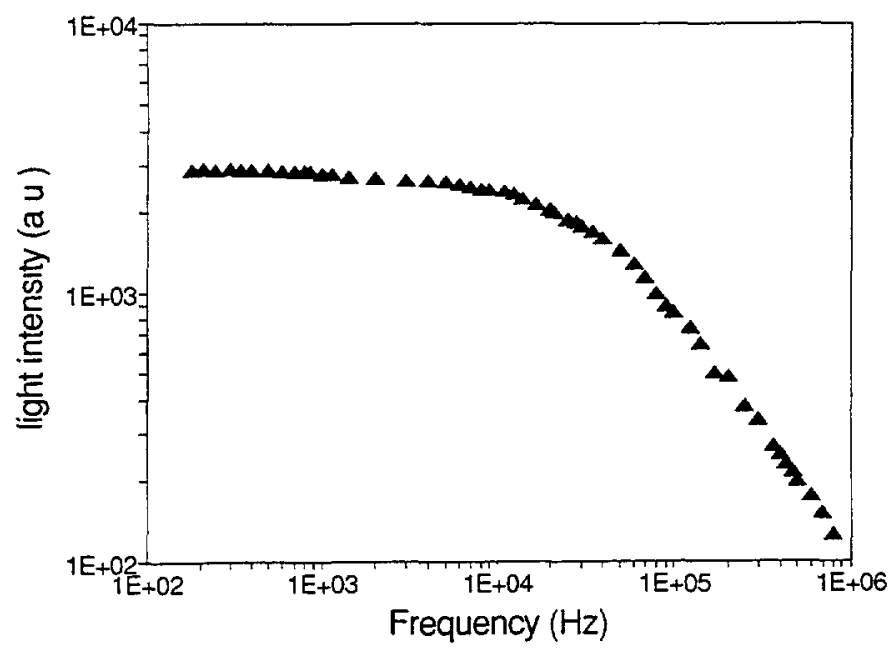

Fig 5 - Log-Log plot of tilt angle modulation in COSC10 Isoleucine showing unambiguously the -1 slope characteristic of the Lorentzian frequency dependence

One then gets the temperature dependence of the plateau of the Lorentzian and of its relaxation frequency in both smectic $A$ and smectic $C^{*}$ phases (Figs 6-7).

These results will be discussed later on but one may already realize that the simplest expectations of equation (7) hold perfectly, at least for our most studied test compound COSC10 Isoleucine

32 Electrical CURRENT MEASUREMENT - We designed an original set-up in order to measure the current in the sample under exactly the same conditions as in optical measurements, $1 \mathrm{e}$ the same temperature and applied voltage in the same cell We took

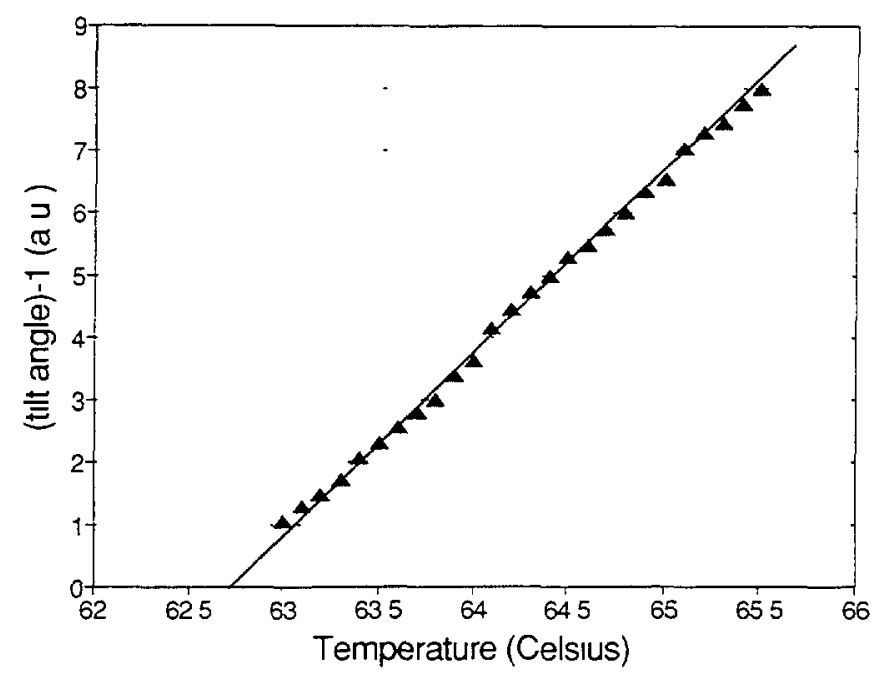

Fig 6 Temperature dependence of the inverse of the tilt angle at $1 \mathrm{kHz}$ in the smectic $\mathrm{A}$ phase of COSC10 Isoleucine 


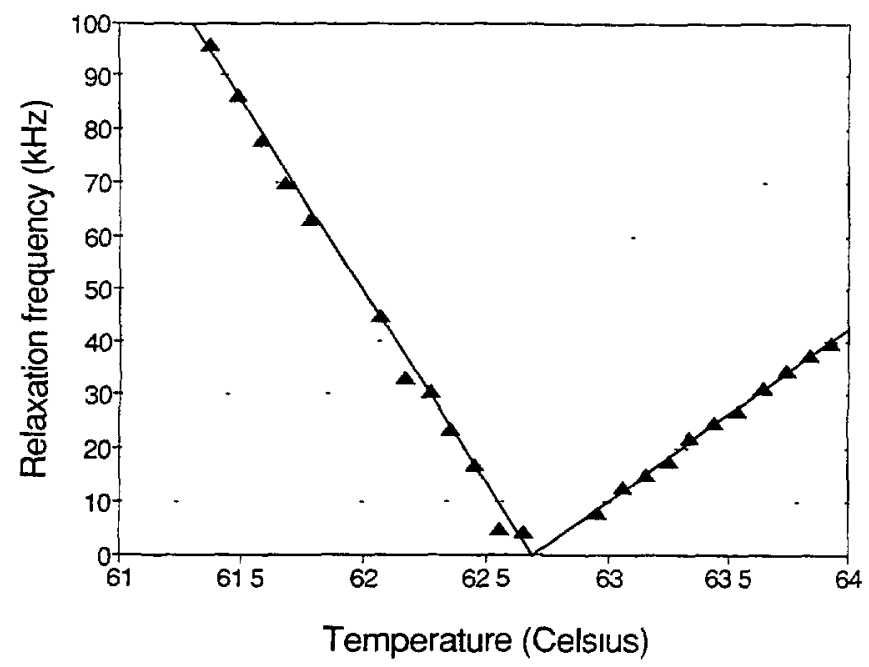

Fig 7 - Temperature dependence of the relaxation frequency in smectic $A$ and smectic $C^{*}$ phase of COSC10 Isoleucine close to the phase transition This shows the expected $1-2$ ratio (see e $\mathrm{g}$ (Eq 7))

advantage of our polarization measurement set-up [22] that uses a current probe (clipped around the electrical connection wire, see Fig 1), which is able to detect a current without adding a load to the impedance of the circuit This one is made of a $1 \mathrm{nF}$ capacitance due to studied sample in series with the $50 \Omega$ resistance of ITO coating on the cell glasses Then the relaxation frequency $1 / 2 \pi R C$ is expected to be about $3 \mathrm{MHz}$ Below this frequency, the measurement of the current yields directly the capacitance of the cell as $l(\omega)=J \omega C(\omega)$ The capacitance is directly proportional to the displacement $D$ (Eq. (10)) in the cell which. in turn, contains a dielectric and an electroclinic component as given in equations (5) and (7). A typical experimental scan is shown in figures 8 and 9.

$$
D=\frac{l(\omega)}{S \omega}=\left(\varepsilon_{\perp}+\frac{\Delta \varepsilon_{\perp}}{1+\jmath \omega \tau}\right) E
$$

where

$$
\begin{gathered}
\varepsilon_{\perp}=\varepsilon_{0}\left(1+\chi_{\propto}+\chi_{\perp}\right) \\
\Delta \varepsilon_{\perp}=\frac{\varepsilon_{0}^{2} \chi_{\perp}^{2} c^{2}}{B_{\perp}^{*}} \\
\tau=\frac{\gamma_{\theta}}{B_{\perp}^{*}}
\end{gathered}
$$

The comparison of the two sets of experiments, $\theta$ and $D$ measurements, allows testing of the coherence of our approach We find that both obey the same temperature and frequency dependence laws 1 e. with a response function $\propto\left[\left(T-T_{c}^{*}\right)+J \omega \gamma_{\theta}\right]^{-1}$ The accuracy of the fit for current curves is lower than for tilt angle scans as there are three adjustable parameters (initial and final slopes together with the relaxation frequency) instead of two amplitude and frequency So, for the determination of the coefficients measured we decided to work on the whole frequency range with the optical measurement only, and to make dielectric measurements below the relaxation frequency with the help of a second capacitor in series 


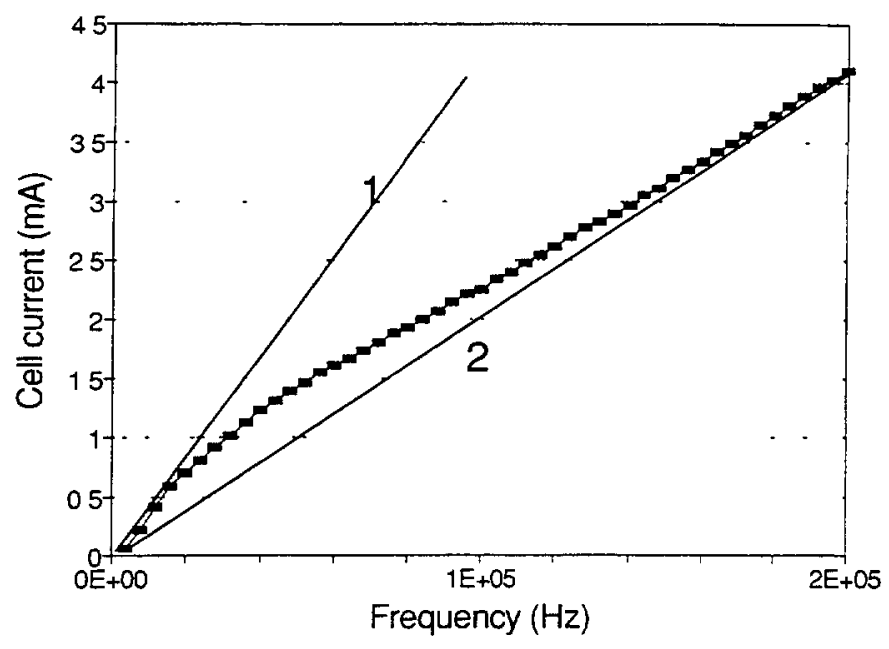

Fig 8 - Current $j \omega C(\omega)$ versus frequency, in COSC10 Isoleucine, showing an initial slope (1) with electrochinic contribution and a relaxation to a smaller one (2) at higher frequency

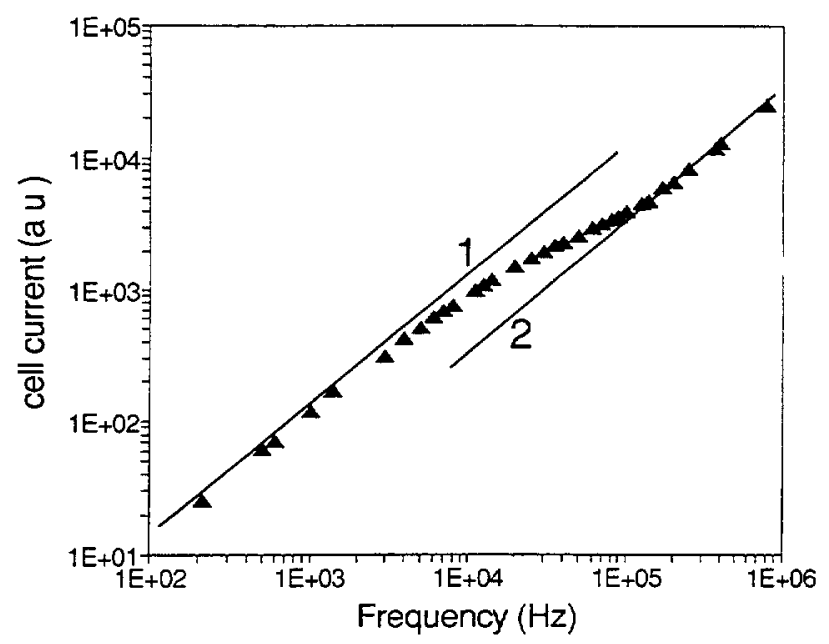

Fig 9 - Doubly logarithmic plot of $j \omega C(\omega)$ in COSC10 Isoleucine showing the relaxatıon at $\approx 50 \mathrm{hHz}$

Wh the cell This allowed us eventually to plot the reduced quantities $\varepsilon_{\mathrm{r} \perp}=D / \varepsilon_{0} E$ versus $\theta / E$ at different temperatures. like in figure 10 , where one gets separately the value of $\chi_{\perp}$ derived from the intercept $\left(1+\chi_{x}+\chi_{\perp}\right)$ of the straight line with the vertical axis, and the reduced electroclinic coefficient [27] $\chi_{\perp} c$ as the slope of the line This shows unambiguously that these two coefficients are temperature independent in the linear regime

\section{Experimental results.}

41 STUDIED COMPOUNDS - We studied three compounds C6 Isoleucine which presents a first order SA-SC ${ }^{\gamma}$ phase transition, COSC10 Isoleucine and $\mathrm{C} 7$ Valine for which the transition was found to be second order 


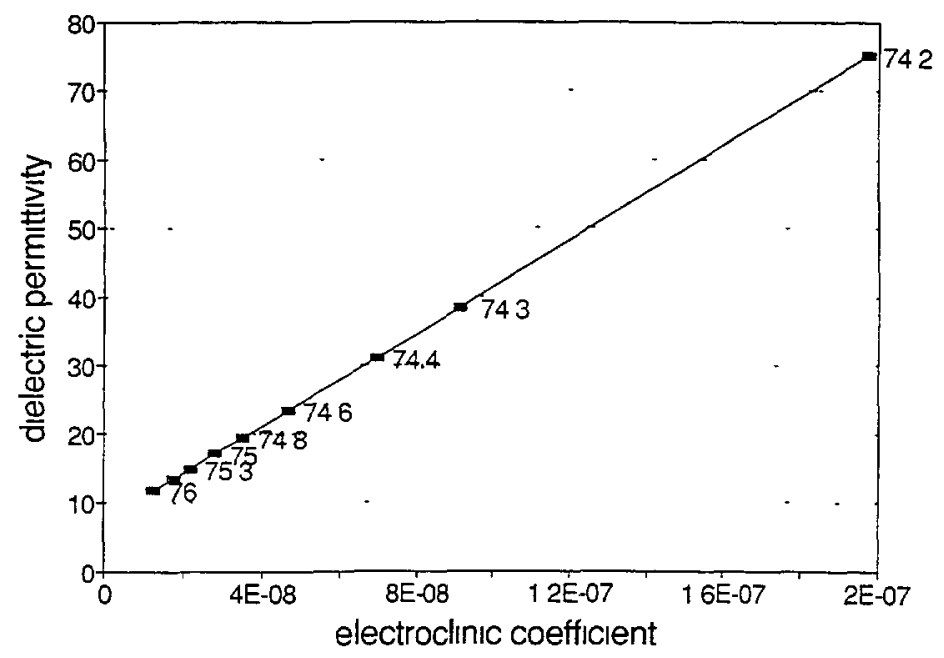

Fig $10-D / \varepsilon_{0} E\left(1 \mathrm{e} \varepsilon_{r_{\perp}}\right.$ ) versus $\theta / E$ (electroclinic coefficient $\varepsilon_{0} \chi_{\perp} c / B_{-}^{*}$ ) in C7 Valine with $15 \mu \mathrm{m}$ thickness at various temperatures (shown inside) and voltages

42 QUANTITATIVE RESUlTS - As previously stated, our experimental procedure allows us to determine in the smectic A phase of a given compound the first coefficients derived from the Landau expansion, namely $\alpha, T_{\mathrm{c}}^{*}, c$ and $\chi_{\perp}$ together with the soft mode viscosity $\gamma_{\theta}$ Their values are given in SI units except for $\varepsilon_{0} \chi_{\perp} c$ which is expressed in $n \mathrm{C} \mathrm{cm}^{-2} \mathrm{rad}^{-1}$ in order to compare it easily with usual $P / \theta$ ratios in smectic $C^{*}$ phase

All our results are to be found in table II

Table II - First coefficients of the Landau expansion (3) and combined expressions for the three studied compounds

\begin{tabular}{|l|c|c|c|}
\hline \multicolumn{1}{|c|}{ Compound } & I & II & III \\
\hline$\chi_{\perp}$ & 5 & 63 & 5 \\
\hline$\varepsilon_{0} \chi_{\perp}\left(\left(\mathrm{nC} \mathrm{cm}^{-2} \mathrm{rad}^{-1}\right)\right.$ & $13 \times 10^{2}$ & $21 \times 10^{2}$ & $30 \times 10^{2}$ \\
\hline$((\mathrm{SI})$ & $29 \times 10^{7}$ & $38 \times 10^{7}$ & $68 \times 10^{7}$ \\
\hline$\alpha\left(N \mathrm{~m}^{-2} \mathrm{~K}^{-1}\right)$ & $43 \times 10^{4}$ & $41 \times 10^{4}$ & $11 \times 10^{5}$ \\
\hline$\Delta T_{\mathrm{c}}=\varepsilon_{0} \chi_{\perp} c^{2} \alpha(\mathrm{K})$ & 11 & 19 & 1.3 \\
\hline$f_{\mathrm{c}}\left(T_{\mathrm{c}}^{*}-1\right)=\alpha / 2 \pi \gamma_{\theta}(\mathrm{kHz})$ & 30 & 100 & 350 \\
\hline$\gamma_{t}($ Poiseulle $)$ & 023 & 0067 & 005 \\
\hline
\end{tabular}

A comment may be made on each of the quantities measured

$-\chi_{\perp}$ is measured with the help of the intercept of $D / \varepsilon_{0} E$ with $\theta / E=0$ axis (see Fig 10) 
Its typical value of 5 implies that $\left(1+\chi_{\infty}+\chi_{\perp}\right) \approx 8$ at low frequencies in the absence of electroclinic coupling, which is quite reasonable

$-\varepsilon_{0} \chi_{\perp} c$, obtained through the slope $\chi_{\perp} c$ of the same curve, has an order of magnitude of 100 and compares well to $P / \theta$ ratios in smectic $C^{*}$ phase This should be used to determine the amount of non lineanty ( 1 e $P_{\mathrm{s}}=\varepsilon_{0} \chi_{\perp} c \theta+\lambda \theta^{2}+$ etc .) in a given compound.

$-\alpha$ (through the slope of $\theta^{-1}$ versus $\left.T\right) \approx 4 \times 10^{4}$ means that $B_{\perp} \approx 10^{7}$ dynes $\mathrm{cm}^{-2}$ far from the transition, as expected for this elastic constant

$-\Delta T_{\mathrm{c}} \approx 1$ to $2 \mathrm{~K}$ compares well again to the difference in transition temperatures between pure compounds and racemic mixtures

- $f_{c}$ obtained from the fit of the Lorentzian $\theta(\omega)$ ranges from 30 to $350 \mathrm{kHz}$ one $\mathrm{K}$ above $T_{\mathrm{c}}$ (1 e more than $1 \mathrm{MHz}$ at higher temperatures for $\mathrm{C} 7$ Valine)

- The value of $\gamma_{\theta}$ determined from $f_{c}$ is rather common for liquid crystals

- No attempt has been made to calculate the critical exponent $\gamma$ in $B_{\perp}$ that is apparently [3.6] close to the mean field value $\gamma=1$ This requires a better understanding of the alignment and layer tilt in samples.

\section{Summary.}

We studied the electroclinic couphing in different compounds, measuring both optical and dielectric properties under the same conditions of temperature, applied electric field and alignment in a given sample We show that one has to obey carefully the prerequisite condition that the applied electric field is low enough in order to work in the linear regime (tilt angle and induced polarization proportional to the field) We measure the first coefficients of the Landau expansion together with the soft mode viscosity and relaxation frequency One finds that the simplest Landau theory of the transition works perfectly with only one temperature dependent coefficient $\left(B_{\perp}^{*}\right)$, the other ones being constant within the smectic $A$ temperature range, in the limits of our experimental accuracy

Then the experiment we describe may be used both for systematic studies in search for better performances of chemical compounds, or from a fundamental point of view to study the different behaviors of the smectic $A$ to smectic $C^{*}$ phase transition.

\section{Appendix.}

When expressing the free energy increase in the smectic A phase, due to the deviations of the order parameter out of its equilibrium value, two cases have to be distinguished, depending on the chirality of the molecules

\section{A1. Achiral molecules.}

The Landau free energy density reads

$$
F=\frac{1}{2} B_{1 / h \ell} Q_{1 /} Q_{h \ell}+\frac{1}{3} \Omega_{i j h \ell m n} Q_{1 /} Q_{h \ell} Q_{m n}+\frac{1}{4} b_{1 j h \ell m n o p} Q_{1 /} Q_{k \ell} Q_{m i n} Q_{u p}
$$

Using the $x, y$ axes in the layer plane and $z$ axis parallel to the layer normal. equation (11) reduces to

$$
\begin{aligned}
& F=\frac{B_{-}}{2 \varepsilon_{4}^{2}}\left[Q_{i=}^{2}+Q_{i=}^{2}\right]+\frac{b}{4 \varepsilon_{a}^{4}}\left[Q_{i=}^{2}+Q_{i=}^{2}\right]^{2}+\frac{1}{2} B^{\prime \prime}\left[\left(Q_{11}-Q_{11}\right)^{2}+4 Q_{i}^{2}\right] \\
& +\Omega\left[\left(Q_{1}-Q_{11}\right)\left(Q_{i=}^{2}-Q_{i=}^{2}\right)-4 Q_{n} Q_{i} Q_{i=}\right]
\end{aligned}
$$


This allows for the distinction of two modes First, the smectic C mode is characterized by the order parameters $Q_{x z}$ and $Q_{y z}$ that are proportional to the parameters defined in equation (1) (namely $Q_{x z}=\varepsilon_{a} \theta_{x}$ and $Q_{y z}=\varepsilon_{a} \theta_{y}$ with $\varepsilon_{a}=\varepsilon_{\|}-\varepsilon_{\perp}$ ) if one forgets about the smectic layers displacement. The biaxial mode, on the other hand, is characterized by the order parameters $Q_{x x}-Q_{y y}$ and $Q_{x y}$ describing the possibility of a new smectic A phase, where the $x$ and $y$ directions should no longer be equivalent [9] These two modes are coupled by the last term of equation (12). The occurrence of a phase transition is determined by the thermal behavior of the quadratic coefficients $B_{\perp}$ and $B^{\prime \prime}$, depending on the one that cancels first. Until now, only $S_{\mathrm{A}}-S_{\mathrm{C}}$ transitions are known for achiral compounds $T$ his can be translated in the mean field approximation by:

$$
B_{\perp}=\alpha\left(T-T_{\mathrm{AC}}\right), \quad \alpha>0, \quad B^{\prime \prime}>0
$$

The $S_{\mathrm{A}}-S_{\mathrm{C}}$ transition seems to be always second order, so that the quartic coefficient should be positive even after the renormalization due to minimization with respect to the (fast) braxial fluctuations

$$
b^{\prime}=b-2 \varepsilon_{\mathfrak{d}}^{4} \Omega^{2} / B^{\prime \prime}>0
$$

\section{A2. Chiral molecules.}

In this case, the absence of inversion center allows new terms in the Landau free energy density representing essentially couplings with an electric polarization $P$ in the layer planes

$$
\begin{aligned}
F^{*}= & F+\frac{\chi_{\imath \jmath}^{-1}}{2 \varepsilon_{0}} P_{\imath} P_{\jmath}+c_{\imath \jmath h} Q_{\imath \jmath} P_{h}+\beta_{\imath \jmath h \ell} Q_{\imath \jmath} P_{h} P_{\ell} \\
& +\lambda_{\imath \jmath k \ell m} Q_{\imath \jmath} Q_{k \ell} P_{m}+h_{\imath \jmath \ell \ell m n} Q_{\imath \jmath} Q_{h \ell} P_{m} P_{n} \\
& +\mu_{\imath \jmath \ell \ell m} Q_{\imath \jmath} P_{k} P_{\ell} P_{m}+\nu_{\imath \jmath k \ell m n o} Q_{l \jmath} Q_{h \ell} Q_{m n} P_{o}
\end{aligned}
$$

$F$ is given in equations (11) and (12) and takes into account the previous order parameters $\left(Q_{12}, Q_{12}\right)$ for the smectic $\mathrm{C}$ mode and $\left(Q_{\mathrm{rr}}-Q_{11}, Q_{\mathrm{n}}\right)$ for the biaxial mode while additional odd coupling terms come in $F^{\times}$Usually the smectic $C$ mode condenses first, leading to a ferroelectric phase Let us note however that a biaxial smectic A phase has been reported recently [9] in a chiral compound, meaning that the biaxial mode would have driven the transition Although defined as a secondary order parameter [8], $P$ represents the amount of permanent molecular dipoles aligned at point $r$ where the free energy density is taken This polarization consists of two contributions, the usual paraelectric one, proportional to any applied electric field and a new one, permanent in the smectic $\mathrm{C}^{*}$ phase, which is proportional at first order to $Q_{i}$

In the $x y=$ frame, equation (13) reduces to

$$
\begin{aligned}
& F^{r}=F+\frac{1}{2 \varepsilon_{0} \chi_{1}}\left[P_{1}^{2}+P_{1}^{2}\right]+\frac{c}{\varepsilon_{a}}\left[Q_{1} P_{1}-Q_{1} P_{1}\right] \\
& +\beta\left[\left(Q_{1}-Q_{1}\right)\left(P_{1}^{2}-P_{1}^{2}\right)+4 Q_{n} P_{1} P_{1}\right] \\
& +\lambda\left[\left(Q_{11}-Q_{11}\right)\left(Q_{12} P_{x}+Q_{x z} P_{1}\right)+2 Q_{x 1}\left(Q_{12} P_{1}-Q_{\mathrm{v}} P_{1}\right)\right] \\
& +\mu\left(Q_{1} P_{1}-Q_{1} P_{1}\right)\left(P_{1}^{2}+P_{1}^{2}\right) \\
& +\nu\left(Q_{1 z} P_{1}-Q_{1 z} P_{1}\right)\left[Q_{i z}^{2}+Q_{i z}^{2}\right] \\
& +f\left[Q_{1=}^{2}+Q_{i=}^{2}\right]\left[P_{1}^{2}+P_{1}^{2}\right]+g\left[Q_{12} P_{1}+Q_{12} P_{1}\right]^{2} \\
& +h\left[Q_{\mathrm{i}} P_{\mathrm{i}}-Q_{\mathrm{i}} P_{\mathrm{l}}\right]^{2}
\end{aligned}
$$


The first line in equation (14) contains all the quadratic terms to be used in the linear electroclinic effect we report on It shows that the in-plane polarization is coupled to the order parameters and that it lies along the $C_{2}$ axis In the smectic $A$ phase close to the transition, one may minimize $F^{*}$ with respect to $P$ and to the biaxial mode, showing that in the smectic $C^{*}$ cybotactic groups a polarization of order $\theta$ and a biaxiality of order $\theta^{2}$ do exist

Then. in first approximation, the remaining terms of equation (14) are of order $\theta^{4}$ leading to a renormalization of the coefficient $b$ of the quartic term in $F$ It has been previously proposed [23,24], but the authors took only into account the last biquadratıc term, that a sign change of the $b$ coefficient is responsible for the observed first order character of the transition with some high polarization compounds $[25,26]$. One should note that the biquadratic term is not specific to the chiral case and that on the contrary there are new antisymmetric terms ( $\mu$ and $\nu$ ) in equation (14) that would induce the sign change of $b$ with chiral compounds only [27]

The gradient terms have not been included in these equations, but it is obvious that a term linear in $\left(\nabla_{\gamma} Q_{k z}-\nabla_{\gamma} Q_{x z}\right)$ leads to the so-called spontaneous splay [28] and another one (the Lifshitz term [29] $Q_{x z} \nabla_{z} Q_{y z}-Q_{y z} \nabla_{z} Q_{x z}$ ) leads to the well known helicoidal structure of smectics $\mathrm{C}^{*}$

\section{A3. Connection to linear electroclinic coupling.}

In the general case, one should use the relation [6]: $Q_{x z}=\varepsilon_{a} \times\left(\theta_{x}+\frac{\partial u}{\partial x}\right)$ linking the tilt angle $\theta_{x}\left(\theta_{y}\right)$ to the order parameter $Q_{x z}\left(Q_{y z}\right)$ and taking into account the layer displacement variable $u$ This will be useful when dealing with more involved problems [10, 30] like Rayleigh scattering due to thermal modes or smectic $\mathrm{C}$-smectic $\mathrm{C}$ critical point In the present experiment, where the tilt angle is forced in the $y$ direction by an electric field applied parallel to $x$, one may forget about this and safely use the linear approximation $Q_{y z}=\varepsilon_{a} \theta$, $Q_{x z}=0$

\section{References}

[1] Meyer R B, Liebert L, Strzelecki L, Keller P, J. Phys Lett 30 (1975) 69

[2] Clark N A. Lagerwall S T, Appl Phys Lett 36 (1980) 899

[3] Garoff S, MEYer R B, Phys Rev Lett. 38 (1977) 848

[4] Martinot-lagarde P, Durand G, J Phys. 42 (1981) 269

[5] Andersson G. Dahl I, Keller P, Kuczynski W, Lagerwall S T, Skarp K and Stebler B, Appl Phys Lett 51 (1987) 640

[6] Dupont L. Thesis 476 Université de Bordeaux I (1990)

[7] DE GENNES (P G), The Physics of Liquid Crystals (Clarendon Oxford Press, Oxford, 1974)

[8] Blinc R, ZeKS B, Phys Rev A 18 (1978) 740

[9] Hiraoka K.. Taguchi A, Ouchi Y, Takezoe H, Fukuda A, Jpn J Appl Phys 29 (1989) L103

[10] Lalanne J R, Buchert J, Destrade C., Nguyen h T, Marcerou J P. Phy's Rev Lett 26 (1989) 3046

[11] Birgeneau R J, Garland C W.. Kortan A R, Litster J D, Meichle M, Ocko B. M, Rosenblatt C, Yu L J, Goodby J . Phys Rev A 27 (1983) 1251

[12] Galerne Y, $J$ Phys 46 (1985) 733.

[13] Benguigut L, Martinoty P, Phys Rev Lett 63 (1989) 774

[14] BAHR Ch, Glenn Brown award lecture 13th I L C C Vancouver (1990)

[15] Glogarova $M$ et al, private communication

[16] Legrand C, Parneix J P, J Phys 51 (1990) 787 
[17] Clark N A., Rieker T P, Phys. Rev A 37 (1988) 1053.

[18] Ouchi Y., Takanishi Y, Takezoe H, Fukuda A., Jap J Appl. Phys. 28 (1989) 2547

[19] Pavel J, Glogarová M, Liquid Crystals in press

[20] Garoff S, Meyer R B, Phys. Rev A 19 (1979) 338

[21] A temperature dependence of the electroclinic coupling coefficient has been nevertheless proposed by $\mathrm{Z}_{\mathrm{L}}$ and C. Rosenblatt, Phys Rev A 39 (1989) 1594.

[22] Nguyen H T, Babeau A., Léon C, Marcerou J P., Destrade C, Soldera A, Guillon D, Skoulios A, Liquid Crystals 9 (1991) 253

[23] Carlsson T, Ž̉̆ǩ̉ B., Filipiè C.. Levstik A, Phys. Rev A 42 (1990) 877

[24] Liu H Y, Huang C. C, Min T, Wand M D, Walba D M, Clark N A, Bahr C, Heppke G, Phys Rev A 40 (1989) 6759

[25] Bahr C, Heppke G., Phys. Rev. A 39 (1989) 5459

[26] Dupont L. Galvan J M, Marcerou J P., Prost J, Ferroelectrics 84 (1988) 317

[27] A careful study of the nature of the phase transition in a series of mixtures of left and right enantiomers presenting a first order transition should be carned out Obviously, the new antisymmetric terms would be zero in the racemic mixture, so the change of the character of the transition from first to second order would give an estimate of the actual influence of these terms Note that such a behavior seems to have been reported in.

Liu H Y, Huang C C, Bahr C., Heppke G, Phys Rev. Lett 61 (1988) 345

[28] Handschy M A, Clark N A., Lagerwall S T., Phys Rev Lett 51 (1983) 471

[29] INDENBOM V L, PIKIN S A, LoGinov E B, Kristallografiya 21 (1976) 1093

[30] Prost J, Defontaines A D, private communication 